\title{
Arterial Spin-Labeling Improves Detection of Intracranial Dural Arteriovenous Fistulas with MRI
}

\author{
(D) S.A. Amukotuwa, (D) M.P. Marks, (D) G. Zaharchuk, (D) F. Calamante, (D) R. Bammer, and DN. Fischbein
}

\begin{abstract}
BACKGROUND AND PURPOSE: Intracranial dural arteriovenous fistulas carry a risk of substantial neurologic complications but can be difficult to detect on structural MR imaging and TOF-MRA. The purpose of this study was to assess the accuracy and added value of 3D pseudocontinuous arterial spin-labeling MR imaging for the detection of these lesions.
\end{abstract}

MATERIALS AND METHODS: This retrospective study included 39 patients with a dural arteriovenous fistula and 117 controls who had undergone both DSA and MR imaging with pseudocontinuous arterial spin-labeling. Two neuroradiologists blinded to the DSA results independently assessed MR imaging with and without pseudocontinuous arterial spin-labeling. They recorded specific signs, including venous arterial spin-labeling signal, and the likelihood of a dural arteriovenous fistula using a 5-point Likert scale. Logistic regression and receiver operating characteristic analyses were performed to determine the accuracy of specific signs and the added value of pseudocontinuous arterial spin-labeling. Interobserver agreement was determined by using $\kappa$ statistics.

RESULTS: Identification of the venous arterial spin-labeling signal had a high sensitivity (94\%) and specificity (88\%) for the presence a dural arteriovenous fistula. Receiver operating characteristic analysis showed significant improvement in diagnostic performance with the addition of pseudocontinuous arterial spin-labeling in comparison with structural MR imaging ( $\Delta$ area under the receiver operating characteristic curve $=0.179$ ) and a trend toward significant improvement in comparison with structural MR imaging with time-of-flight MRA ( $\Delta$ area under the receiver operating characteristic curve $=0.043$ ). Interobserver agreement for the presence of a dural arteriovenous fistula improved substantially and was almost perfect with the addition of pseudocontinuous arterial spin-labeling $(\kappa=0.92)$.

CONCLUSIONS: Venous arterial spin-labeling signal has high sensitivity and specificity for the presence of a dural arteriovenous fistula, and the addition of pseudocontinuous arterial spin-labeling increases confidence in the diagnosis of this entity on MR imaging.

ABBREVIATIONS: $\mathrm{ASL}=$ arterial spin-labeling; $\mathrm{AUC}=$ area under the ROC curve; DAVF $=$ dural arteriovenous fistula; $\mathrm{NCH}=$ nodular and/or curvilinear hyperintensities; NPV = negative predictive value; $\mathrm{PCASL}=$ pseudocontinuous ASL; PPV = positive predictive value; $\mathrm{ROC}=$ receiver operating characteristic; $\mathrm{sMRI}=$ structural MRI

D ural arteriovenous fistulas (DAVFs) are intracranial arteriovenous shunting lesions characterized by direct connection between dural arteries and either a dural sinus or cortical vein. ${ }^{1,2}$ The consequent increase in blood flow and pressure in draining veins can lead to complications, including cerebral edema, ischemia, and hemorrhage. ${ }^{2}$ The criterion standard for the diagnosis

Received April 20, 2017; accepted after revision December 26.

From the Department of Radiology (S.A.A., M.P.M., G.Z., R.B., N.F.), Stanford University, Stanford, California; and Florey Department of Neuroscience and Mental Health (S.A.A., F.C.), University of Melbourne, Melbourne, Victoria, Australia.

Drs Amukotuwa and Bammer are supported by National Institutes of Health ( $\mathrm{Na}$ tional Institute of Biomedical Imaging and Bioengineering) grants 3R01EB00271106S1 and 5R21EB021029. Dr Calamante is supported by the National Health and Medical Research Council of Australia, grant APP1117724.

Please address correspondence to Shalini A. Amukotuwa, MB, BS, Stanford University, Stanford, California; and Florey Department of Neuroscience and Mental Health, University of Melbourne, Melbourne, VIC, Australia 3052; e-mail: samukotuwa@gmail.com of a DAVF, catheter-based DSA, is invasive, uses ionizing radiation, and carries a small-but-significant risk of permanent neurologic injury. ${ }^{3}$

The clinical presentation of DAVFs is nonspecific and broad, ranging from asymptomatic to pulsatile tinnitus, seizures, altered mental status, and/or intracranial hemorrhage. ${ }^{4}$ In some of these patients, an alternative etiology for the presentation is not identified by standard noninvasive imaging methods, and there remains clinical suspicion for a DAVF. A sensitive noninvasive imaging method for the detection of DAVFs would help avoid exposing such patients to the risks (and cost) of diagnostic DSA. 3D TOFMRA, time-resolved contrast-enhanced MRA, and CTA tech-

\footnotetext{
-- Indicates open access to non-subscribers at www.ajnr.org

三 Indicates article with supplemental on-line tables.

http://dx.doi.org/10.3174/ajnr.A5570
}

AJNR Am J Neuroradiol 39:669-77 Apr 2018 www.ajnr.org 
niques have been shown to have a high sensitivity and specificity for the identification of DAVFs. ${ }^{5-10}$ The diagnosis of DAVFs in daily practice on these conventional imaging studies remains a challenge, however. Unlike AVMs, which often have a conspicuous nidus surrounded by brain tissue, DAVFs often manifest only subtle findings on structural imaging. ${ }^{4,11}$ An easily applicable imaging method that increases the conspicuity of DAVFs would therefore be valuable.

Arterial spin-labeling (ASL) is a completely noninvasive MR imaging technique that is now widely available on clinical MR imaging scanners. ASL signal is not observed in cerebral veins under normal conditions due to T1 decay during capillary transit and exchange with the extravascular space of labeled blood-water protons. ${ }^{11,12}$ In conditions with arteriovenous shunting such as DAVFs, rapid transit of labeled blood directly from feeding arteries to draining veins results in high signal in venous structures. ${ }^{11,12}$ This venous ASL signal is a conspicuous finding that can alert the reader to the presence of shunting. Conventional sequences can then be more closely scrutinized for structural signs of a DAVF. Preliminary studies indicate that ASL improves the detection of small intracranial shunting lesions and increases the reader's diagnostic confidence. ${ }^{11}$ These prior investigations, however, included AVMs and only a few DAVFs. ${ }^{1,12}$

The purpose of this case-control study was to assess the value of an MR imaging protocol augmented with 3D pseudocontinuous ASL (pCASL) for the detection of DAVFs and to determine whether reader accuracy and confidence are improved over MR imaging with conventional structural sequences and TOF-MRA.

\section{MATERIALS AND METHODS Patient Population}

This retrospective study was approved by our institutional review board.

Thirty-nine patients ( 15 females, 24 males, 8 -86 years of age; mean age, 55 years) with a DAVF and 117 controls ( 51 females, 66 males, 4 months to 80 years of age; mean age, 50 years) who underwent both DSA and MR imaging with 3D pCASL at our institution between June 1, 2009, (pCASL was used in routine brain MR imaging protocols from this date) and September 30, 2014, were included. To develop our study population, we searched reports of consecutive cerebral DSAs performed during this time for the terms "DAVF," "dural AVF," "arteriovenous fistula," "fistula," and "carotid cavernous." This search yielded 792 patients: 139 consecutive patients with a DAVF and 653 patients with no DAVF.

Patients with DAVFs. Fifty-six of 139 patients with a DAVF diagnosed on DSA also had an MR imaging with 3D pCASL. One patient was excluded due to markedly motion-degraded images; and 16, due to angiographically complete treatment of the fistula before the MR imaging. Of the 39 patients included in the study, MR imaging and DSA were performed before any treatment of the fistula in 26 patients and following partial treatment (with DSA evidence of residual shunting) in 13. Fistula Cognard grades ${ }^{13}$ were the following: I $(n=10), \operatorname{IIa}(n=7), \operatorname{IIb}(n=7), \mathrm{II} \mathrm{a}+\mathrm{b}(n=$ $8)$, III $(n=6)$, and IV $(n=1)$. Fistula locations were the following: transverse and/or sigmoid sinus $(n=14)$, marginal sinus $(n=2)$, superior sagittal sinus $(n=3)$, torcula herophili $(n=1)$, vein of Galen $(n=3)$, sphenoparietal sinus $(n=1)$, cavernous sinus $(n=8)$, cortical vein $(n=6$, two infratentorial), and tentorium cerebelli $(n=1)$. Clinical presentations were the following: intracranial hemorrhage $(n=15)$, pulsatile tinnitus $(n=9)$, chemosis/proptosis $(n=6)$, cranial nerve VI palsy $(n=2)$, seizures $(n=2)$, dementia $(n=1)$, and follow-up of previous dural venous sinus thrombosis $(n=2)$ and meningioma resection $(n=2)$.

Controls. Six hundred fifty-three patients who had undergone DSA due to a clinical presentation (eg, pulsatile tinnitus or intracranial hemorrhage of unclear origin) potentially attributable to an intracranial vascular malformation did not have a DAVF. Patients with an AVM (which is also associated with arteriovenous shunting) on DSA were excluded. Two hundred nineteen of these patients had an MR imaging with pCASL, and 117 of these patients (3 times the number of cases) were randomly selected as controls.

In patients with multiple imaging studies, the DSA and MR imaging examinations temporally closest to each other were selected (mean time interval, 46 days for the control group and 57 days for the DAVF group).

\section{Imaging Methods}

In 68 subjects ( 16 patients, 52 controls), MRIs were performed on a 3 T scanner (Discovery 750w; GE Healthcare, Milwaukee, Wisconsin); and in 88 subjects ( 23 patients and 65 controls), on a $1.5 \mathrm{~T}$ scanner (Signa HDx; GE Healthcare).

3D pCASL imaging (TR/TE, 4800/9.5 ms [1.5T], 5000/10.5 ms [3T]; 24-cm FOV) with background suppression was implemented using a labeling period of $1500 \mathrm{~ms}$, a postlabel delay of $2000 \mathrm{~ms}$, the labeling plane at the level of the foramen magnum, and a segmented 3D stack-of-spirals FSE readout (spiral arms: 6 [1.5T]; $8[3 \mathrm{~T}]$ ). In-plane spatial resolution was $3-4 \mathrm{~mm}$, and through-plane spatial resolution was $4-6 \mathrm{~mm}$, yielding $24-40$ slices. Scan time was 3 minutes 30 seconds -6 minutes. No vascular crusher gradients were used. This acquisition complies with the consensus recommendations of the ASL community. ${ }^{14}$ The presence of venous ASL signal was assessed on the label-control subtraction images and required no postprocessing.

3D multislab TOF-MRA (1.5T: TR/TE, 30/2.9 ms; FOV, 22 cm; matrix, $320 \times 192 ; 1.4-\mathrm{mm}$ slice thickness; 3T: TR/TE, 22/2.5 $\mathrm{ms} ; \mathrm{FOV}=24 \mathrm{~cm}$; matrix $=512 \times 288 ; 1.2-\mathrm{mm}$ slice thickness $)$ was performed in 147 patients ( 36 patients and 111 controls).

All $156 \mathrm{MR}$ imaging examinations also included the following sequences: sagittal T1-weighted (1.5T: TR/TE, 600/15 ms; 3T: TR/ TE/TI, 1800/6.7/920 ms) and axial T2-weighted FSE (TR/TE, 4800/84 ms [1.5T]; 4000/100 ms [3T]); T2*-weighted gradient recalled-echo (TR/TE, 600/30 ms [1.5T]; 600/15 ms [3T]); T2weighted FLAIR (TR/TE/TI, 8802/110/2200 ms [1.5T]; 9000/143/ $2300 \mathrm{~ms}$ [3T]); and diffusion-weighted ( $b=1000 \mathrm{~s} / \mathrm{mm}^{2}$; TR/TE, $6000 / 70 \mathrm{~ms}[1.5 \mathrm{~T}], 5000 / 83 \mathrm{~ms}[3 \mathrm{~T}])$ imaging performed with a 24-cm FOV, 5-mm slice thickness.

Catheter-based DSA for the clinical work-up of the DAVF was performed by 1 of 3 interventional neuroradiologists (with 5, 12, and $>20$ years' experience respectively) in a dedicated biplane neuroangiography suite (Axiom Artis; Siemens, Erlangen, Germany). Imaging included, at minimum, anteroposterior and lat- 

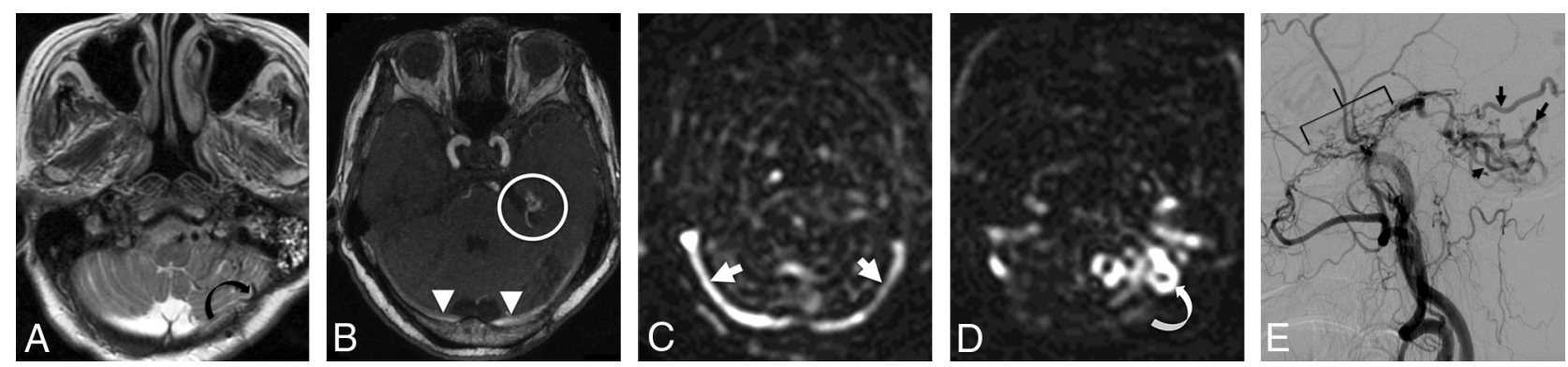

FIG 1. A 79-year-old woman presenting with ataxia. A, T2-weighted imaging demonstrates tortuous and ectatic pial veins (curved black arrow) along the inferior surface of the left cerebellar hemisphere. $B$, MIP TOF-MRA image demonstrates nodular and curvilinear hyperintensities immediately superior to the left petrous apex, representing a left tentorial fistula (white circle) and high signal in the transverse sinuses (white arrowheads). C, PCASL image shows venous ASL signal in the transverse sinuses (white arrows) due to shunting. D, More inferiorly, venous ASL signal is seen in draining pial veins (white curved arrow). E, DSA image following left external carotid artery injection confirms a Cognard type IV left tentorial DAVF (bracket) with a middle meningeal artery supply and drainage directly into ectatic cerebellar cortical veins (black arrows).

eral views obtained following selective injection with iodinated contrast medium (iohexol, Omnipaque 300; GE Healthcare, Piscataway, New Jersey) of the internal carotid, external carotid, and/or vertebral artery supplying the DAVF.

CTA was not included in this evaluation because it is not routinely performed for the evaluation of suspected DAVFs at our institution and it was therefore inconsistently available in the patients in the study population.

\section{Image Interpretation}

MR Imaging. MRIs were independently reviewed in random order by 2 neuroradiologists (with 20 years' and 5 years' postfellowship experience) blinded to the clinical data and DSA findings. Review of nonenhanced CT performed before MR imaging was not permitted because it was unavailable in many patients with DAVFs $(n=31)$.

The readers rated the likelihood of a DAVF on a 5-point Likert scale ( 1 , very unlikely; 2 , unlikely; 3 , equally likely and unlikely; 4 , likely; and 5, very likely) based on sequences presented to them. The reads were performed in 2 separate sittings, with and without pCASL, 2 months apart to negate the effects of memory and learning:

First Sitting. pCASL was omitted.

Step 1. Structural sequences (T1- and T2-weighted imaging, FLAIR, and gradient recalled-echo) were reviewed first to establish the baseline accuracy of MR imaging for identification of DAVFs. We recorded the presence or absence of each of the following signs: intracranial hemorrhage; white matter T2 hyperintensity with mass effect (edema); abnormal vessels (abnormal clusters of flow voids, serpiginous and/or dilated leptomeningeal or medullary vessels, and/or venous pouches) (Fig $1 A$ ); and enlarged (reader's subjective impression) dural venous sinuses. DAVFs in the cavernous sinus may produce characteristic features suggestive of this diagnosis; therefore, we specifically recorded these signs: cavernous sinus enlargement, superior ophthalmic vein enlargement, proptosis, and/or orbital edema. The overall likelihood of a DAVF was then rated on the 5-point Likert scale.

Step 2. In the 147 patients in whom TOF-MRA was performed, source images and MIPs were assessed next. The following signs were recorded as present or absent: hyperintense (signal higher than that of suppressed background tissue and similar to that within arteries) dural venous sinus (Fig $1 B$ ) or cortical vein; nodular and/or curvilinear hyperintense structures ${ }^{5}(\mathrm{NCH})$ adjacent to a dural venous sinus, representing the fistula itself (Fig $1 B$ ); increased number or size of arteries near a dural venous sinus; and/or enlargement of external carotid artery branches. ${ }^{5,6}$ The overall likelihood of a DAVF based on TOF-MRA and structural features was rated on the 5-point Likert scale.

Second Sitting. pCASL was first reviewed in conjunction with anatomic sequences. The presence or absence of venous ASL signal, defined as the presence of high signal intensity on pCASL images within a dural venous sinus (Fig $1 C$ ), deep venous structure, and/or cortical vein (Fig 1D) was recorded. TOF-MRA (source and MIP images) was subsequently reviewed, and the overall likelihood of a DAVF based on all sequences was rated.

The presence of a DAVF on angiography was considered the criterion standard. When there was discordance between the findings on MR imaging and angiography, the DSA and MR imaging were reviewed, in consensus, by the 2 neuroradiologists and an experienced neurointerventional radiologist.

\section{Statistical Analysis}

All statistical analyses were performed using SPSS (SPSS Statistics 24.0.0.1, 64 bit; IBM, Armonk, New York) and MedCalc Statistical Software, Version 17.2, 64 bit (MedCalc Software, Mariakerke, Belgium).

To test the added value of pCASL, we performed sensitivity and specificity analyses (individual reader and pooled) for the following: 1) conventional structural MR imaging (sMRI) alone; 2) structural MR imaging and TOF-MRA (sMRI/MRA); 3) structural MR imaging, TOF-MRA, and pCASL (sMRI/MRA/pCASL) using receiver operating characteristic (ROC) analyses by nonparametric estimation of the area-under-the-ROC-curve (AUC) and subsequent pair-wise comparisons using the DeLong algorithm.

Sensitivity, specificity, positive predictive value (PPV), and negative predictive value (NPV) for individual imaging features were computed using the readers' classification tables, with the reference standard being the presence or absence of a DAVF. The likelihood of a DAVF (on DSA) based on the identification of an 
Table 1: Individual imaging features_-univariate binary logistic regression analysis on DSA and interreader agreement

\begin{tabular}{|c|c|c|c|c|c|c|c|c|c|}
\hline Imaging Feature & OR & SE & $\begin{array}{l}\text { Wald } \\
\text { Test }\end{array}$ & $P$ & $\begin{array}{l}\text { Sensitivity } \\
(95 \% \mathrm{Cl})(\%)\end{array}$ & $\begin{array}{l}\text { Specificity } \\
(95 \% \mathrm{Cl})(\%)\end{array}$ & $\begin{array}{c}\text { PPV } \\
(95 \% \mathrm{Cl})(\%)\end{array}$ & $\begin{array}{c}\text { NPV } \\
(95 \% \text { CI) (\%) }\end{array}$ & $\begin{array}{c}\text { Interobserver } \\
\text { Agreement } \\
\text { (к) }(95 \% \mathrm{CI})\end{array}$ \\
\hline \multicolumn{10}{|l|}{ Structural MRI } \\
\hline $\mathrm{ICH}$ & 0.18 & 1.32 & 37.84 & .001 & $37.2(26.5-47.9)$ & $23.1(17.7-28.5)$ & $13.9(9.2-18.6)$ & $52.4(43.0-62.1)$ & $0.99(0.97-1.00)$ \\
\hline Vasogenic edema or gliosis & 0.36 & 1.37 & 10.59 & .001 & 20.8 (11.7-58.1) & $58.1(51.8-64.4)$ & $14.0(7.7-20.4)$ & $69.0(62.6-75.5)$ & $1.00(1.00-1.00)$ \\
\hline Abnormal vessels & 5.57 & 1.32 & 37.24 & .001 & $59.0(48.1-69.9)$ & 79.5 (74.3-84.7) & $48.9(38.8-59.0)$ & $85.3(80.6-90.0)$ & $0.73(0.67-0.79)$ \\
\hline Enlarged sinus & 2.58 & 1.36 & 9.50 & .002 & $31.2(20.8-41.5)$ & $79.9(73.9-85.8)$ & $40.7(28.1-53.2)$ & $72.4(66.1-78.7)$ & $0.69(0.61-0.76)$ \\
\hline Enlarged SOV & 3.68 & 1.62 & 7.36 & .007 & $12.7(5.3-20.0)$ & $96.2(93.7-98.6)$ & $52.6(30.2-75.1)$ & $76.5(71.7-81.4)$ & $0.83(0.74-0.93)$ \\
\hline Cavernous sinus enlargement & 34.80 & 2.14 & 21.85 & .001 & $23.1(13.7-32.4)$ & $99.2(98.0-100.3)$ & $90.0(76.9-103.2)$ & 79.5 (74.8-84.1) & $0.89(0.82-0.97)$ \\
\hline Orbital edema/proptosis & 12.26 & 2.23 & 10.38 & .001 & $10.3(3.5-17.0)$ & $99.2(98.0-100.3)$ & $80.0(55.2-104.8)$ & $76.8(72.1-81.6)$ & $1.00(1.00-1.00)$ \\
\hline \multicolumn{10}{|l|}{ Time-of-flight MRA } \\
\hline $\mathrm{NCH}$ & 114.70 & 1.88 & 56.75 & .001 & $61.1(49.9-72.4)$ & $98.7(97.1-100.2)$ & $93.6(86.6-100.6)$ & $88.7(84.7-92.6)$ & $0.87(0.82-0.93)$ \\
\hline Abnormal vessels & 6.00 & 1.34 & 37.28 & .001 & $59.7(48.4-71.1)$ & $80.2(74.9-85.4)$ & $49.4(38.9-59.9)$ & $86.0(81.3-90.7)$ & $0.38(0.30-0.46)$ \\
\hline Venous signal & 20.75 & 1.47 & 60.96 & .001 & 87.5 (79.9-95.1) & $74.8(69.1-80.5)$ & $52.9(44.0-61.9)$ & $94.9(91.6-98.1)$ & $0.87(0.83-0.91)$ \\
\hline Enlarged extracranial arteries & 17.81 & 1.41 & 70.98 & .001 & $62.5(51.3-73.7)$ & 91.4 (87.8-95.1) & $70.3(59.1-81.5)$ & 88.3 (84.1-92.4) & $0.76(0.69-0.83)$ \\
\hline \multicolumn{10}{|l|}{ ASL } \\
\hline Venous ASL signal & 103.20 & 1.65 & 84.95 & .001 & $93.6(88.2-99.0)$ & 87.6 (83.4-91.8) & $71.5(62.8-80.3)$ & 97.6 (95.6-99.7) & $0.94(0.9-0.97)$ \\
\hline
\end{tabular}

Note:-SOV indicates superior ophthalmic vein; $\mathrm{SE}$, standard error; $\mathrm{ICH}$, intracerebral hemorrhage.
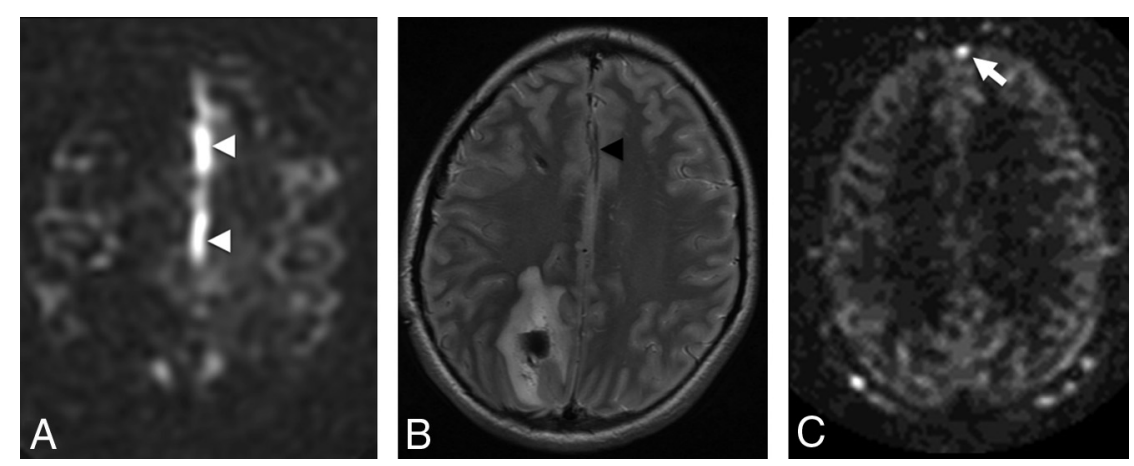

FIG 2. False-positive venous ASL signal in 2 patients. A, ASL signal in the $A 3$ branches of the anterior cerebral arteries (white arrowheads) was mistaken for venous ASL signal in a 15-year-old male patient who presented with a right parietal parenchymal hematoma. $B$, T2-weighted images show localization of this signal to the anterior cerebral arteries (black arrowhead). C, ASL signal in the anterior aspect of the superior sagittal sinus in a 70-year-old man with subarachnoid hemorrhage (white arrow). This patient had no evidence of a DAVF or shunting on DSA.

individual imaging feature was determined using univariate binary logistic regression. A likelihood ratio test between multivariate binary logistic regression models was performed to determine whether the addition of the venous ASL signal feature yielded a stronger association with the presence of a DAVF than structural features alone or structural-plus-TOF-MRA features.

Interobserver agreement between readers was assessed using the Cohen $\kappa$ statistic for specific imaging features and a linear weighted $\kappa$ statistic for the likelihood of a DAVF. A $\kappa$ of at least 0.61 (substantial agreement) was made a prerequisite to pool reader scores for subsequent analysis.

\section{RESULTS}

\section{Individual Imaging Features}

Venous ASL signal had the highest sensitivity (94\%; 95\%, CI $88 \%-99 \%)$ and NPV (98\%; 95\% CI, 96\%-100\%) for DAVFs of all the investigated specific features (Table 1).Venous ASL signal and other signs of DAVFs were not visible even on post hoc review of 2 false-negative cases. Both were low-flow DAVFs ( 1 incidentally detected tentorial fistula in a patient undergoing DSA for evaluation of aneurysmal subarachnoid hemorrhage and 1 low-flow cavernous sinus DAVF).

Nodular and/or curvilinear hyperintensities on TOF-MRA (Fig $1 B$ ) had a much lower sensitivity (61\%; 95\% CI, 50\%-72\%). Of the 36 patients with a fistula who had undergone TOF-MRA, the fistula was located above the TOFMRA slab in 3 on post hoc analysis. Of the 33 patients whose fistula was located within the TOF-MRA slab, $\mathrm{NCH}$ were not identified in 9 (reader 1 ) and 13 (reader 2) patients, and they were absent in 7 patients $(21 \%)$ on post hoc review. Three were Cognard III fistulas with a single feeding artery.

Univariate binary logistic regression analyses found extremely high odds ratios for both venous ASL signal (103) and $\mathrm{NCH}$ (114), reflecting their high specificity. There were a number of false-positives for venous ASL signal in our cohort; therefore, its specificity (88\%; 95\% CI, 83\%-92\%) was lower than that of $\mathrm{NCH}(99 \%$; $95 \%$ CI, 97\%-100\%). On post hoc review of these 16 false-positive cases, cortical vein ASL signal was evident in 4 and dural venous sinus ASL signal was seen in 11. A definite cause for this ASL signal such as an AVM, hypervascular tumor, or subacute stroke could not be discerned on review of these patients' clinical histories and follow-up imaging, including DSAs. In 3 patients, the cortical vein ASL signal was observed immediately adjacent to a parenchymal hematoma, and we hypothesize that the hematoma may have compressed and obscured (on DSA and conventional MR imaging) a small parenchymal AVM. The other most likely explanation for venous ASL signal in these cases, as well as in 7 of the patients with dural sinus ASL signal, is seizure incited by parenchymal hematoma. In the 4 remaining patients, mild ASL signal in the anterior superior sagittal sinus (Fig $2 C$ ) was not associated with parenchymal hematoma, and this is likely a normal variant.

There may have been a small underlying AVM in each of these cases, obscured and compressed by hematoma, hence occult on DSA and structural MR imaging. Alternatively, subclinical seizures incited by hematoma would also account for the venous ASL signal.

Arterial transit artifact ${ }^{15}$ was mistaken for venous ASL signal in 1 control (Fig 2A). Venous hyperintensity on TOF-MRA had a lower specificity (75\%; 95\% CI, 69\%-80\%) than venous ASL sig- 
nal due to a greater number of false-positives (26 for reader 1 and 30 for reader 2).

Structural imaging signs of a fistula were of high specificity but low sensitivity and NPV. Although signs of a cavernous sinus DAVF had high specificity and odds ratios, fistulas in this location were too sparsely represented in our study cohort to draw meaningful conclusions related to these findings.

Agreement between the readers was almost perfect $(\kappa>0.81)$ for all individual features except 3 TOF-MRA parameters: enlarged sinus, enlarged extracranial arteries, and abnormal vessels (Table 1). Only fair agreement $(\kappa>0.21)$ was reached on the latter.

\section{Overall Assessment}

The ROC plots (Fig 3), their corresponding AUCs, and the substantial differences in the AUCs demonstrate improvement in diagnostic performance for the detection of DAVFs with the addition of pCASL (Table 2). Diagnostic performance significantly improved with the addition of TOF-MRA to sMRI (pooled $\triangle$ AUC, $0.14 ; P<.01$ ). The addition of pCASL significantly improved diagnostic performance over sMRI (pooled $\triangle$ AUC, 0.179; $P<.01)$. Improvement in diagnostic performance with the addition of pCASL to $\mathrm{sMRI/TOF-MRA} \mathrm{reached} \mathrm{statistical} \mathrm{significance}$ for reader 2 but not reader 1 (whose excellent diagnostic performance on sMRI and TOF-MRA left little room for improvement). When reader performance was pooled, a trend toward improvement was seen (with only $11 \%$ probability that the observed improvement in the detection of DAVFs was due to chance).

These ROC analysis findings were corroborated by the multivariate binary logistic regression models indicating a significant contribution to diagnostic performance from the addition of TOF-MRA and pCASL features to sMRI (Table 3 and On-line Tables 1 and 2). If we applied a $P=.5$ classification threshold (Table 4), sensitivity was poor for sMRI (33\%; 95\% CI, 22\%$44 \%$ ), improved if TOF-MRA was added (sensitivity, $76 \%$; $95 \% \mathrm{CI}, 66 \%-86 \%$ ), and increased further with pCASL (sensitivity, 89\%; 95\% CI, 81\%-96\%) at comparably high specificity (On-line Table 2). When used in combination, sMRI, MRA, and pCASL yielded a high NPV of 96.4\% (95\% CI, 93.9\%$98.9 \%$ ), which is crucial if MR imaging is to be used as a screening tool.

Incremental improvement in reader confidence in the presence or absence of a DAVF with the addition of PCASL is illustrated in Fig 4.

Interobserver agreement was good for structural imaging alone $(\kappa=0.62 ; 95 \% \mathrm{CI},-1.0-1.0)$ and improved with the addition of TOF-MRA $(\kappa=0.72 ; 95 \%$ CI, $-1.0-1.0)$. It improved substantially and was almost perfect with the addition of pCASL $(\kappa=0.92 ; 95 \% \mathrm{CI},-0.23-1.0)$.

In 4 cases, a DAVF was present on DSA but was considered unlikely on MR imaging until pCASL was reviewed. In 1 case, the fistula was located outside the TOF-MRA slab. In the remaining 3 cases, the fistula was not visible on TOF-MRA despite being located within the slab, and other MRA signs were also absent. Two of these cases were partially treated fistulas with low residual flow on DSA.
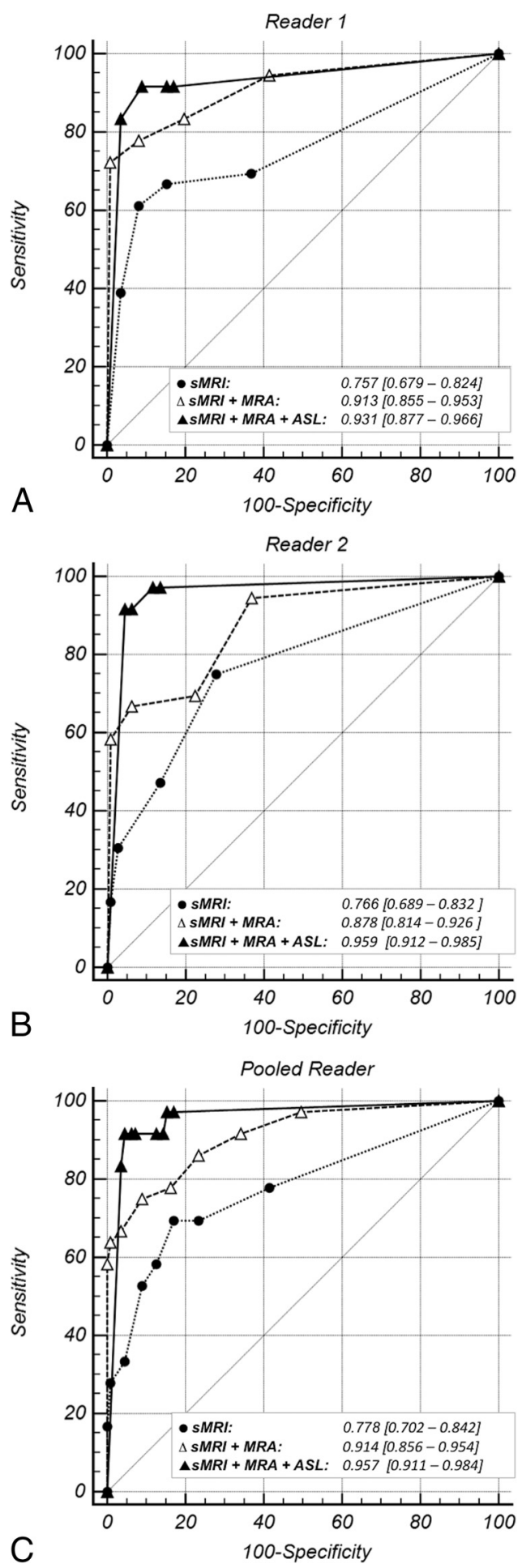

FIG 3. ROC curves for each reader's individual diagnostic performance $(A$ and $B)$ and their pooled diagnostic performance $(C)$ with each of the following: structural MR imaging (dotted line), structural MR imaging and TOF-MRA (sMRI + MRA, dashed line), and structural MR imaging with TOF-MRA and PCASL (sMRI + MRA + pCASL, solid line). The light gray diagonal is the line of no discrimination. The triangle and dot symbols on the curve indicate true-positive rate/ false-positive rate pairs computed at different discrimination thresholds. With the addition of TOF-MRA and then PCASL to structural MR imaging, the ROC curve becomes more well-rounded with an incrementally higher AUC. This indicates increased diagnostic sensitivity for detection of a DAVF at a set specificity. 


\begin{tabular}{|c|c|c|c|c|c|}
\hline Reader, Diagnostic Instrument & $\Delta$ AUC & SE & $95 \% \mathrm{Cl}$ & $z$ & $P$ \\
\hline \multicolumn{6}{|l|}{ Reader 1} \\
\hline sMRI vs sMRI + MRA & 0.156 & 0.046 & $0.066-0.247$ & 3.381 & $<.01$ \\
\hline sMRI vs sMRI + MRA + pCASL & 0.174 & 0.047 & $0.082-0.266$ & 3.701 & $<.01$ \\
\hline$s M R I+M R A$ vs sMRI+MRA + pCASL & 0.018 & 0.028 & $-0.036-0.072$ & 0.644 & .52 \\
\hline \multicolumn{6}{|l|}{ Reader 2} \\
\hline sMRI vs sMRI + MRA & 0.112 & 0.044 & $0.027-0.197$ & 2.581 & $<.01$ \\
\hline sMRI vs sMRI + MRA + pCASL & 0.193 & 0.043 & $0.108-0.277$ & 4.475 & $<.01$ \\
\hline$s M R I+M R A$ vs sMRI + MRA + pCASL & 0.081 & 0.030 & $0.022-0.140$ & 2.675 & $<.01$ \\
\hline \multicolumn{6}{|l|}{ Pooled Readers $1+2$} \\
\hline sMRI vs sMRI + MRA & 0.136 & 0.042 & $0.053-0.219$ & 3.208 & $<.01$ \\
\hline sMRI vs sMRI + MRA + pCASL & 0.179 & 0.045 & $0.091-0.267$ & 3.965 & $<.01$ \\
\hline$s M R I+M R A$ vs sMRI + MRA + pCASL & 0.043 & 0.027 & $-0.009-0.100$ & 1.615 & .11 \\
\hline
\end{tabular}

Table 3: Pair-wise likelihood ratio tests for the multivariate models

\begin{tabular}{lrrrr}
\hline \multicolumn{1}{c}{ Reader, Diagnostic Instrument } & $\mathbf{- 2 L L}$ & $\boldsymbol{\chi}^{2}$ & $\boldsymbol{P}$ & $\boldsymbol{\Delta d \boldsymbol { d f }}$ \\
\hline sMRI & 260.18 & & & \\
sMRI + MRA & 117.09 & & & \\
sMRI + MRA + ASL & 84.23 & & & \\
(sMRI) vs (sMRI + MRA) & & 143.09 & $<.001^{\mathrm{a}}$ & 4 \\
(sMRI + MRA) vs (sMRI + MRA + ASL) & & 32.86 & $<.001^{\mathrm{a}}$ & 1 \\
(sMRI) vs (sMRI + MRA + ASL) & & 175.95 & $<.001^{\mathrm{a}}$ & 8 \\
\hline
\end{tabular}

Note:-LL indicates log likelihood.

a Significance $(P<.001$ ) - that is, that the null hypothesis (difference in $-2 \mathrm{LLS}=0$ ) is rejected and that the -2 LLs are different.

\section{DISCUSSION}

This case-control study confirmed that venous ASL signal has a high sensitivity and specificity and an even higher NPV than previously reported ${ }^{11}$ for detecting the presence of an intracranial DAVF. Identification of venous ASL signal improves the diagnostic accuracy and increases confidence in the presence or absence of a DAVF. We have previously shown that MR imaging with pCASL can be used to accurately determine the grade of a DAVF. ${ }^{16}$ In this study, we investigated the clinical utility of pCASL for detection of DAVFs.

A reliable noninvasive technique for detection of DAVFs can improve patient triage, expedite referral for treatment, and decrease the number of DSAs a patient with a DAVF undergoes by allowing diagnostic and therapeutic angiography to be performed at the same session. Perfect or almost perfect sensitivity and NPV are requisite, given that failure to detect a DAVF has a greater potential for adverse consequences than a false-positive. Unfortunately, conventional structural MR imaging is unreliable for the diagnosis of DAVF as seen in this study. TOF-MRA was also less sensitive than previously reported. For example, nodular/curvilinear hyperintensities have been previously reported to be $100 \%$ sensitive, ${ }^{5}$ but these were absent even on post hoc review in $21 \%$ of DAVFs. Three were high-grade fistulas with a single feeding artery draining directly into a nonectatic cortical vein that would not be expected to manifest as nodular/curvilinear hyperintensities.

Venous ASL signal had a higher sensitivity for the presence of a DAVF than structural or TOF-MRA signs. There were only 2 cases in which the venous ASL signal was absent. The high conspicuity of the venous ASL signal against the relatively flat background of normal ASL signal makes it easy to detect when present. Nulling of static tissue signal by background suppression and subtraction of control from label images produces a high contrast-to- noise ratio with only labeled blood giving rise to signal on pCASL images. ${ }^{15,16}$ One hundred percent of the voxel is occupied by labeled blood in a draining vein in comparison with $2 \%-5 \%$ in a tissue voxel. ${ }^{16}$ There is also greater T1 decay of signal of labeled blood by the time it reaches the tissue in comparison with labeled blood shunted directly into venous structures. Consequently, there is a large contrast differential between the draining veins of a DAVF and adjacent brain parenchyma. ${ }^{16}$ Absence of ASL signal in arteries under normal conditions also enhances the conspicuity of the venous ASL signal. Conversely, high signal is seen in normal arteries on both TOF-MRA and contrast-enhanced MRA. A hyperintense cortical vein may therefore be dismissed or misclassified as an artery on these sequences.

pCASL is likely to be particularly beneficial to less experienced readers: The junior reader in this study had a lower sensitivity for detection of DAVFs on SMRI and TOF-MRA and demonstrated greater improvement in diagnostic performance following the addition of PCASL. The readers' confidence and agreement regarding the presence or absence of a DAVF also improved following review of pCASL. Interreader agreement for venous ASL signal was higher than for TOF-MRA signs. These findings suggest that venous ASL signal is not only easier to detect but can also be interpreted with greater certainty. Even when present, findings on structural sequences and TOF-MRA are often subtle and equivocal, requiring scrutiny of images and high diagnostic skill to identify and interpret; this has relevance in routine clinical practice, in which there is a broad range of reader experience levels and more likelihood that an unsuspected positive case will be mixed in with many negative cases. The high conspicuity of venous ASL signal may also enable detection of unsuspected DAVFs.

Venous ASL signal also had a high specificity for the presence of a DAVF. Theoretically, high signal is absent in venous structures on PCASL under normal conditions due to T1 decay of labeled blood during the time it takes to pass through the tissue capillary bed. ${ }^{11,12}$ The shorter time that labeled blood takes to reach veins when shunted directly precludes significant $\mathrm{T} 1$ decay. Venous ASL signal is therefore seen in conditions with arteriovenous shunting, including hypervascular tumors, seizures, stroke, and AVMs. ${ }^{11,12}$ False-positive cases of venous ASL signal in this study were likely due to one of these alternative causes of shunting, such as seizure or an occult AVM obscured by hematoma. Four control patients with mild ASL signal in the anterior aspect of the superior sagittal sinus lacked a plausible alternative cause of shunting. The authors have observed mild ASL signal in 
Table 4: Classification tables for the multivariate binary logistic regression models ${ }^{\mathrm{a}}$

\begin{tabular}{lcccc}
\multicolumn{1}{c}{ MRI Sequences } & Sensitivity (95\% Cl) (\%) & Specificity (95\% Cl) (\%) & PPV (95\% CI) (\%) & NPV (95\% CI) (\%) \\
\hline Structural MRI alone & $32.9(22.3-43.5)$ & $97.0(94.8-99.2)$ & $78.1(63.8-92.5)$ & $81.7(77.1-86.2)$ \\
Structural MRI and TOF-MRA & $75.7(65.7-85.8)$ & $98.7(97.1-100.2)$ & $94.6(88.8-100.5)$ & $92.8(89.5-96.1)$ \\
Structural MRI, TOF-MRA and PCASL & $88.6(81.1-96.0)$ & $96.4(93.9-98.9)$ & $88.5(81.1-96.0)$ & $96.4(93.9-98.9)$ \\
\hline
\end{tabular}

${ }^{a}$ Classification table generated for a probability value of $P=.05$ for each of the 3 multivariate models.

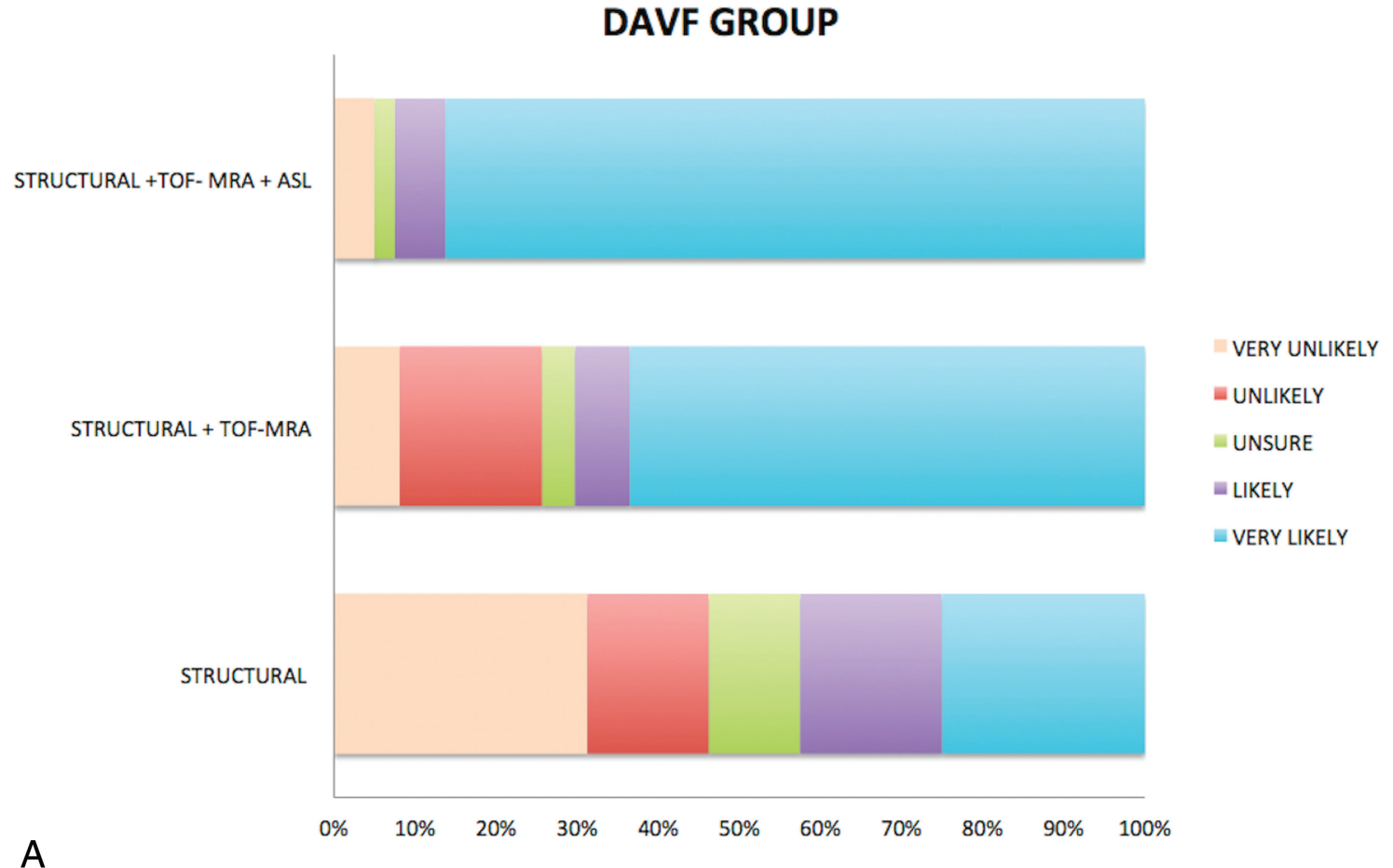

CONTROL GROUP

STRUCTURAL +TOF- MRA + ASL

STRUCTURAL + TOF-MRA

B

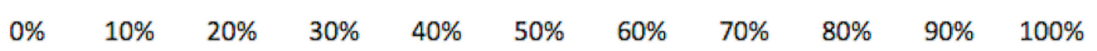

FIG 4. Distribution of Likert scale scores for patients with $(A)$ and without $(B)$ a DAVF on structural MR imaging alone, structural MR imaging with TOF-MRA (sMRI/MRA), and structural MR imaging with both TOF-MRA and PCASL (sMRI/MRA/pCASL). A, In the DAVF group, there is a marked incremental increase in reader accuracy and confidence in the presence of a DAVF-with a higher percentage considered "very likely" to have a DAVF- with the addition of pCASL. B, In the control group, reader accuracy and confidence in the absence of a DAVF decrease with the addition of TOF-MRA to SMRI due to a high number of false-positives for venous hyperintensity on TOF-MRA. Reader certainty as to the absence of a fistula increased (and was highest) following review of PCASL. 
this location in other patients without a shunting lesion. Readers should be cognizant of this potential normal variant.

Another advantage of pCASL is whole-brain coverage in a reasonable scan time. Despite technologic advances such as 3T and parallel imaging, coverage of TOF-MRA often does not extend to the vertex due to clinical time constraints. DAVFs located superior to the imaged slabs may therefore be missed.

Venous ASL signal was absent in 2 low-flow DAVFs, likely due to an inadequate volume of shunted blood to produce perceptible signal in draining veins. Two other low-flow fistulas were detected on pCASL but missed on TOF-MRA (likely due to signal saturation). There were too few ${ }^{4}$ low-flow DAVFs in this study to draw meaningful conclusions regarding this population. Long-label long-delay pCASL may allow detection of these low-flow fistulas, because more labeled blood would be present in draining veins at a later imaging. ${ }^{15}$

Another potential pitfall of pCASL is high signal in arterial structures in conditions with delayed transit of labeled blood such as steno-occlusive disease. ${ }^{11,15}$ Cognizance of this arterial transit $\operatorname{artifact}^{15}$ (Fig 2A) and careful cross-reference with other sequences are necessary to avoid false-positives.

Contrast-enhanced MRA has been reported to be accurate for the detection of DAVFs. ${ }^{5,7,17}$ The diagnosis requires identification of early venous filling, which is likely more challenging than identification of the conspicuous venous ASL sign, given the compromise between spatial and temporal resolution required to perform clinical contrast-enhanced MRA; even with highly constrained reconstruction techniques, spatial and temporal resolution is much lower than that of DSA. ${ }^{18}$ Contrast-enhanced MRA also requires administration of gadolinium-based contrast, adding to the cost. Gadolinium is also associated with a risk of anaphylaxis, nephrogenic systemic fibrosis, and deposition. Other advanced techniques for detection and characterization of DAVFs such as $4 \mathrm{D}$ ASL-based $\mathrm{MRA}^{18}$ are still research tools not available for clinical use.

pCASL is also available on both $1.5 \mathrm{~T}$ and $3 \mathrm{~T}$ clinical units and is feasible within the constraints of everyday clinical practice. Given its utility for detection of intracranial shunting, we recommend its routine inclusion in the MR imaging protocol for patients with intracranial hemorrhage and pulsatile tinnitus. It should also be added to the protocol for those patients with clinical or noninvasive imaging findings raising suspicion for a DAVF.

The major limitation of this study is the small number of lowflow DAVFs, which may potentially be occult on pCASL. Its retrospective nature also limited us to reviewing routine MR images obtained at our institution. Prospective enrollment would enable inclusion and comparative assessment of CTA, contrast-enhanced MRA, and alterative ASL techniques (eg, long-label longdelay, which may be more sensitive for shunting).

\section{CONCLUSIONS}

Venous ASL signal has high sensitivity and specificity for the presence of a DAVF; hence, its identification increases confidence in the presence or absence of a DAVF on noninvasive imaging. It is also a highly conspicuous finding that is easy to detect and has the potential to increase diagnostic performance, particularly of less experienced readers. False-positives can occur and are likely due to alternative causes of intracranial shunting. False-negatives also occur, with some small and low-flow fistulas occult on MR imaging with PCASL so that sensitivity is imperfect compared with the criterion standard of DSA. We therefore do not advocate replacing DSA for the screening of DAVFs at this stage if clinical suspicion of a DAVF is high, but MR imaging with pCASL can help refine triage and expedite management by increasing confidence in the diagnosis.

\section{ACKNOWLEDGMENTS}

The authors would like to thank Dr Jarrett Rosenberg for his feedback on the statistical analyses.

Disclosures: Shalini A. Amukotuwa-RELATED: Grant: National Institute of Biomedical Imaging and Bioengineering, Comments: grant numbers 5R21EB021029, 3R01EB002711-06S1.* Fernando Calamante-RELATED: Grant: National Health and Medical Research Council of Australia; UNRELATED: Grant: Australian Research Council; Payment for Lectures Including Service on Speakers Bureaus: Siemens.* Greg Zaharchuk-UNRELATED: Grants/Grants Pending: GE Healthcare, National Institutes of Health. Roland Bammer-RELATED: Grant: National Institute of Biomedical Imaging and Bioengineering, Comments: grant numbers 5R21EB021029, 3R01EB002711-06S1*; Support for Travel to Meetings for the Study or Other Purposes: National Institutes of Health (National Institute of Biomedical Imaging and Bioengineering). * *Money paid to the institution.

\section{REFERENCES}

1. Newton TH, Cronqvist S. Involvement of dural arteries in intracranial arteriovenous malformations. Radiology 1969;93:1071-78 CrossRef Medline

2. Cognard C, Casasco A, Toevi M, et al. Dural arteriovenous fistulas as a cause of intracranial hypertension due to impairment of cranial venous outflow. J Neurol Neurosurg Psychiatry 1998;65:308-16 CrossRef Medline

3. Cloft HJ, Joseph GJ, Dion JE. Risk of cerebral angiography in patients with subarachnoid hemorrhage, cerebral aneurysm, and arteriovenous malformation: a meta-analysis. Stroke 1999;30:317-20 CrossRef Medline

4. Miller TR, Gandhi D. Intrancranial dural arteriovenous fistulae: clinical presentation and management strategies. Stroke 2015;46: 2017-25 CrossRef Medline

5. Noguchi K, Melhem ER, Kanazawa T, et al. Intracranial dural arteriovenous fistulas: evaluation with combined 3D time-of-flight $M R$ angiography and MR digital subtraction angiography. AJR Am J Roentgenol 2004;182:183-90 CrossRef Medline

6. Kwon BJ, Han MH, Kang HS, et al. MR imaging findings of intracranial dural arteriovenous fistulas: relations with venous drainage patterns. AJNR Am J Neuroradiol 2005;26:2500-07 Medline

7. Meckel S, Maier M, Ruiz DS, et al. MR angiography of dural arteriovenous fistulas: diagnosis and follow-up after treatment using a time-resolved 3D contrast-enhanced technique. AJNR Am J Neuroradiol 2007;28:877-84 Medline

8. Farb RI, Agid R, Willinsky RA, et al. Cranial dural arteriovenous fistula: diagnosis and classification with time-resolved MR angiography at 3T. AJNR Am J Neuroradiol 2009;30:1546-51 CrossRef Medline

9. Nishimura S, Hirai T, Sasao A, et al. Evaluation of dural arteriovenous fistulas with $4 \mathrm{D}$ contrast-enhanced $\mathrm{MR}$ angiography at $3 \mathrm{~T}$. AJNR Am J Neuroradiol 2010;31:80-85 CrossRef Medline

10. Brouwer PA, Bosman T, van Walderveen MA, et al. Dynamic 320section CT angiography in cranial arteriovenous shunting lesions. AJNR Am J Neuroradiol 2010;31:767-70 CrossRef Medline

11. Le TT, Fischbein NJ, André JB, et al. Identification of venous signal on arterial spin labeling improves diagnosis of dural arteriovenous fistulas and small arteriovenous malformations. AJNR Am J Neuroradiol 2012;33:61-68 CrossRef Medline 
12. Wolf RL, Wang J, Detre JA, et al. Arteriovenous shunt visualization in arteriovenous malformations with arterial spin-labeling MR imaging. AJNR Am J Neuroradiol 2008;29:681-87 CrossRef Medline

13. Cognard C, Gobin YP, Pierot L, et al. Cerebral dural arteriovenous fistulas: clinical and angiographic correlation with a revised classification of venous drainage. Radiology 1995;194:671-80 CrossRef Medline

14. Alsop DC, Detre JA, Golay X, et al. Recommended implementation of arterial spin-labeled perfusion MRI for clinical applications: a consensus of the ISMRM perfusion study group and the European consortium for ASL in dementia. Magn Reson Med 2015;73:102-16 CrossRef Medline

15. Amukotuwa SA, Yu C, Zaharchuk G. 3D pseudocontinuous arterial spin labeling in routine clinical practice: a review of clinically sig- nificant artifacts. J Magn Reson Imaging 2016;43:11-27 CrossRef Medline

16. Amukotuwa SA, Heit JJ, Marks MP, et al. Detection of cortical venous drainage and determination of the Borden type of dural arteriovenous fistula by means of 3D pseudocontinuous arterial spin-labeling MRI. AJR Am J Roentgenol 2016;207:163-69 CrossRef Medline

17. Clark Z, Johnson KM, Wu Y, et al. Accelerated time-resolved contrast-enhanced magnetic resonance angiography of dural arteriovenous fistulas using highly constrained reconstruction of sparse cerebrovascular data sets. Invest Radiol 2016;51:365-71 CrossRef Medline

18. Iryo $\mathrm{Y}$, Hirai $\mathrm{T}$, Kai $Y$, et al. Intracranial dural arteriovenous fistulas: evaluation with 3-T four-dimensional MR angiography using arterial spin labeling. Radiology 2014;271:193-99 CrossRef Medline 